IdeAs

Idées d'Amériques

$6 \mid 2015$

Migrer dans les Amériques

\title{
Whom We Shall Welcome : L'évolution des politiques migratoires américaines depuis le Hart-Celler Act de 1965
}

\section{Bénédicte Deschamps}

\section{(2) OpenEdition Journals}

Édition électronique

URL : https://journals.openedition.org/ideas/1290

DOI : $10.4000 /$ ideas. 1290

ISSN : 1950-5701

Éditeur

Institut des Amériques

Référence électronique

Bénédicte Deschamps, "Whom We Shall Welcome : L'évolution des politiques migratoires américaines depuis le Hart-Celler Act de 1965 », IdeAs [En ligne], 6 | 2015, mis en ligne le 18 décembre 2015,

consulté le 19 octobre 2022. URL : http://journals.openedition.org/ideas/1290 ; DOI : https://doi.org/ 10.4000/ideas. 1290

Ce document a été généré automatiquement le 19 octobre 2022.

\section{(c) (i) (9)}

Creative Commons - Attribution - Pas d'Utilisation Commerciale - Pas de Modification 4.0 International - CC BY-NC-ND 4.0

https://creativecommons.org/licenses/by-nc-nd/4.0/ 


\title{
Whom We Shall Welcome : L'évolution des politiques migratoires américaines depuis le Hart-Celler Act de 1965
}

\author{
Bénédicte Deschamps
}

1 Titre d'un célèbre rapport commandé par le président Harry Truman pour évaluer l'efficacité de la loi sur l'immigration de 1952 (Mc Carran-Walter Act), Qui nous accueillerons (Whom we Shall Welcome) soulève une question toujours d'actualité aux États-Unis ${ }^{1}$ (United States. President's Commission, 1953). À l'époque, la commission chargée de rédiger ledit rapport ne manqua pas de souligner les limites et les incohérences de la législation existante. Elle avait été adoptée contre le veto de Truman et maintenait les quotas sur l'origine nationale des immigrés établis en 1921 et 1924 dans le but de réduire drastiquement les flux «indésirables » provenant d'Europe de l'Est et du Sud. Or, dans les années 1950, les enjeux idéologiques étaient de taille. Comment pouvait-on encore conserver des mesures inspirées par les théories eugénistes, et refuser ainsi de tirer les enseignements de la Seconde Guerre mondiale ? Comment renforcer les liens des États-Unis avec des pays européens alliés dans la guerre froide contre le communisme, quand ceux-ci - telle l'Italie - faisaient partie des nationalités jugées indésirables? Le rapport répondit à ces interrogations en concluant que le Mc Carran Act incarnait "des politiques et des principes" «imprudents et préjudiciables pour la nation" et qu'il devait être "reconsidéré et révisé de fond en comble» (United States. President's Commission, 1953: 263). Il proposa aussi une série d'amendements qui servirent de fondement à la réforme de 1965.

2 L'Immigration and Nationality Act de 1965 (Hart-Celler Act) fut le fruit de cette refonte et constitua, on le sait, un tournant majeur dans la politique migratoire des États-Unis. L'un des artisans de la loi, le représentant démocrate Emanuel Celler, avait à cœur de défendre un texte qui devait, comme le déclara le président Lyndon B. Johnson, 
corriger " un profond et douloureux défaut dans la structure de la justice américaine » (Johnson L.B., 1965). Certes, les forces conservatrices résistèrent à la réforme et le texte finalement adopté s'avéra moins libéral que prévu (Marinari M., 2014). Toutefois, à l'heure des luttes pour les droits civiques, les membres du Congrès qui se battirent pour abolir les quotas étaient sincères dans leur démarche et connaissaient le poids symbolique des amendements qu'ils soutenaient (Chin G.J., 2015). Ce n'est pas un hasard si la loi fut signée par Johnson au pied de la Statue de la Liberté, rappelant ainsi la longue tradition d'accueil qui avait fait la réputation des États-Unis. Alors que la guerre du Vietnam faisait rage, le président expliquait qu'il était temps de reconnaître que les soldats américains tombés au combat pouvaient s'appeler Zelinko ou Mariano sans que «ni les ennemis qui les tuaient ni les gens dont ils défendaient l'indépendance " ne cherchent à savoir "d'où ils venaient, ou d'où venaient leurs parents » (Johnson L.B., 1965). En somme, il était devenu indispensable pour les ÉtatsUnis de mettre en accord leur politique migratoire à la fois avec leurs objectifs diplomatiques et avec l'évolution des rapports interraciaux de leur société.

Outre l'élimination des anciens quotas, le Hart Celler Act faisait apparaître un nouveau système, en partie déjà introduit en 1952, celui des catégories préférentielles. Celles-ci favorisaient le rapprochement familial, le recrutement de travailleurs qualifiés ou nécessaires à l'économie américaine et l'accueil de réfugiés originaires de pays communistes ou moyen-orientaux ${ }^{2}$. La loi fixait également des plafonds de 170000 entrées pour l'Hémisphère Est (Europe, Asie, Afrique) et de 120000 pour les Amériques. En signant le Hart-Celler Act, Johnson affirma que ce texte n'était " pas révolutionnaire " et n'affecterait "pas la vie de millions de personnes» (Johnson L.B., 1965). Tous les historiens s'accordent aujourd'hui à souligner combien cette prophétie était erronée. Le nouveau cadre établi par le Hart-Celler Act, sur lequel repose encore toute la législation actuellement en vigueur, changea la donne à bien des égards. Survenue alors même que se modifiait la situation économique et démographique des pays latinoaméricains, la réforme eut des "conséquences imprévues", qui transformèrent le visage des États-Unis. Après 1965, en raison de facteurs multiples, dont la loi sur l'immigration, on observa une mutation radicale de l'origine des flux migratoires, ces derniers provenant désormais en majorité d'Amérique latine (surtout le Mexique, le Salvador et Cuba) et d'Asie (essentiellement la Chine, l'Inde et les Philippines) et non plus de l'Europe. Ce résultat est assez paradoxal dans la mesure où, pour la première fois, la loi fixait précisément un plafond pour l'immigration latino-américaine (Massey D. et Pren K.A., 2012) (voir l'article de Jim Cohen dans ce dossier).

Deux réformes majeures suivirent la loi de 1965 : l'Immigration Reform and Control Act votée en 1986 et l'Illegal Immigration Reform and Immigrant Responsibility Act, adoptée dix ans plus tard. Toutes deux, ainsi que celles qui suivirent, s'attaquaient au problème de l'immigration illégale, sans proposer de refonte à long terme de la politique migratoire générale du pays. La première, qui se voulait un compromis entre générosité et répression, permit la régularisation de certains immigrés (2,7 millions) (Cooper B. et O'Neil K., 2005: 3) tout en prévoyant des sanctions pour les employeurs embauchant des travailleurs sans papiers valides. La seconde durcit les mesures punitives, à un moment où l'opinion publique se polarisait, comme les partis, sur la question des « immigrés illégaux ». La loi prévoyait notamment un renforcement de la sécurité à la frontière avec le Mexique ( 5000 agents supplémentaires, plus 12 millions de dollars pour un mur de 15,5 km de San Diego vers l'est), et une refonte plus sévère des procédures d'expulsion. Malgré la présence d'une large population hispanique, qui 
continue d'augmenter et constitue un électorat déterminant dans certains États, toute la législation votée depuis 1996 tend vers plus de contrôle, d'arrestations et de reconductions à la frontière (Ngai M., 2014). Le traumatisme du 11 septembre 2001 a évidemment laissé des traces. Le Patriot Act, ainsi que la création du Department of Homeland Security (DHS) ont conduit à toujours plus de fermeté, au nom de la sécurité nationale, mais au risque d'associer dans l'esprit des citoyens américains immigration et terrorisme. Certes, les mouvements de défense des immigrés ont contribué à faire avorter le Border Protection, Antiterrorism and Illegal Immigration Control Act de 2005 qui tendait à « criminaliser » encore davantage les étrangers en situation irrégulière, mais le problème de ces derniers n'est pas réglé (Feldman D., 2013). D'autant que les États, notamment l'Arizona, compliquent les choses en votant leurs propres lois sur l'immigration, créant ainsi un dispositif à couches multiples. Au fil de lois comme le Secure Fence Act de 2006, le mur séparant le Mexique des États-Unis a aussi pris une importance politique et symbolique grandissante. Plus il s'allonge et s'épaissit, plus les passeurs se professionnalisent, et plus le trafic humain enrichit les organisations criminelles. Franchir ce rempart illégalement est devenu si dangereux que 307 décès ont été enregistrés en 2014 (chiffre inférieur à celui des années précédentes (U.S. Customs and Border Protection, 2014 ; Cohen J., 2011).

5 La politique migratoire américaine témoigne du dilemme des États-Unis, tiraillés entre des exigences sécuritaires accrues et les besoins d'une économie dépendant en partie de la main-d'œuvre irrégulière. Dès son élection, le président Barack Obama avait promis une réforme censée "réparer » un "système d'immigration en panne » (Obama B., 2014). Mais ses efforts se sont heurtés aux résistances des franges les plus conservatrices du Congrès, notamment au Tea Party. Non seulement l'administration Obama n'a pas pu tenir ses promesses envers les immigrés « non autorisés », mais elle a accéléré le rythme des arrestations et des expulsions. Le nombre de personnes reconduites à la frontière au cours de l'année 2014 se montait ainsi à 315 943, selon le Department of Homeland Security (DHS, 2014). Après le double échec du Dream Act (Development, Relief, and Education for Alien Minors Act), repoussé par le Sénat en 2006 et 2010, qui proposait de régler le problème des mineurs entrés illégalement sur le territoire avec leurs parents et qui, bien que scolarisés et intégrés dans la société, ne peuvent accéder aux mêmes droits que les citoyens américains, le président passe à l'action en juin 2012, en pleine période de campagne électorale. Des mesures provisoires sont prises pour les seuls mineurs en situation irrégulière (Deferred Action for Childhood Arrivals, DACA). L'année suivante, un groupe de sénateurs composé de quatre républicains et quatre démocrates (le "Gang of Eight») a proposé un nouveau projet de loi soutenu par Obama (Border Security, Economic Opportunity, and Immigration Modernization Act) qui n'a pas abouti, et qui ne satisfaisait ni les restrictionnistes, ni les associations de défense des droits des immigrés. Face à cette impasse législative, Obama a décidé de recourir à un décret présidentiel, à la fin de 2014, pour avancer sur certains points en attendant que le Congrès ne statue à nouveau sur ces questions. Il s'est fixé alors trois objectifs : renforcer le contrôle des frontières, faciliter l'immigration légale des travailleurs qualifiés, et éviter l'expulsion à certains immigrés en situation irrégulière (résidant depuis plus de cinq ans aux États-Unis, au casier vierge et acceptant de s'inscrire sur les registres du DHS et de payer des impôts) (Obama B., 2014). En somme, rien qui ne puisse contenter les associations soutenant la cause des sans-papiers, ni résoudre le problème de la présence non autorisée de onze millions de personnes que le républicain Donald Trump proposait récemment de renvoyer 
massivement mais « humainement » dans leur pays (Wang Y., 2015), comme on l'avait fait pour les Mexicains en 1954, lors de la très controversée " Operation Wetback »"

Début octobre 2015, en réponse à la crise des réfugiés syriens, Obama a lancé un appel à la générosité des Américains, et s'est engagé à en accueillir 10000 (chiffre très limité) pour participer à la solidarité internationale (Miller J., 2015). Aujourd'hui comme hier, les forces d'inclusion et de rejet des immigrés continuent de s'opposer mais il semble bien que la tradition d'asile économique, politique et religieux, portée haut par la Statue de la Liberté, ait fait long feu, vaincue par la crise économique, le retour en force du nativisme et la menace terroriste.

\section{BIBLIOGRAPHIE}

Chin, Gabriel J., "Were the Immigration and Nationality Act Amendments of 1965 Anti-Racist?", In Gabriel J. Chin, Rose Cuison Villazor, The Immigration and Nationality Act of 1965: Legislating a New America, Cambridge, Cambridge University Press, 2015, p. 11-59.

Cohen, James, « De Bush à Obama, la réforme bloquée du contrôle de l'immigration », Amérique Latine Histoire et Mémoire. Les Cahiers ALHIM, n²2, 2011, http://alhim.revues.org/4049, consulté le 29 sept. 2015.

Cooper, Betsy et Kevin O'Neil, “Lessons From the Immigration Reform and Control Act of 1986", In MPI Policy Brief, n³, Août 2005, p. 3, http://www.migrationpolicy.org/research/lessonsimmigration-reform-and-control-act-1986, consulté le 15 décembre 2015.

Feldman, David, « Whose Immigration Reform? », La Revue des droits de l'homme, n4, 2013, http:// revdh.revues.org/349, consulté le 27 sept. 2015

President Lyndon B. Johnson's Remarks at the Signing of the Immigration Bill, Liberty Island, New York, October 3, 1965, Public Papers of the Presidents of the United States: Lyndon B. Johnson, 1965. Volume II, entry 546, p. 1037-1040, Washington, D. C.: Government Printing Office, 1966.

Marinari, Maddalena, “Americans Must Show Justice in Immigration Policies Too": The Passage of the 1965 Immigration Act”, Journal of Policy History, Vol. 26, n², 2014, p. 219-245.

Massey, Douglas S. et Karen A. Pren, "Unintended Consequences of US Immigration Policy: Explaining the Post-1965 Surge from Latin America," Population and Development Review, vol. 38, $\mathrm{n}^{\circ} 1$, mars 2012, p. 1-29.

Miller, Joshua, “\#AidRefugees: Heeding the President's Call to Take Action”, 6 octobre 2015, https://www.whitehouse.gov/blog/2015/10/06/aidrefugees-heeding-presidents-call-take-action.

Motomura, Hiroshi, Immigration Outside the Law, Oxford, Oxford University Press, 2014.

Ngai, Mae M., "Undocumented migration to the United States : a History" In Lois Ann Lorentzen (dir.), Hidden Lives and Human Rights in the United States. Understanding the Controversies and Tragedies of Undocumented Immigration, Santa Barbara, CA, Praeger, 2014, p. 2-23. 
Obama, Barack, "Remarks by the President in Address to the Nation on Immigration", 20 novembre 2014, https://www.whitehouse.gov/issues/immigration/immigration-action\#, consulté le 3 octobre 2015 .

U.S. Customs and Border Protection, "US Border patrol Stats FY2014 sector profile", p. 1 https:// www.cbp.gov/sites/default/files/documents/USBP\%20Stats\%20FY2014\%20sector\%20profile.pdf, consulté le 19 août 2015.

U.S. Immigration and Customs Enforcement, « FY 2014 ICE Immigration Removals », http:// www.ice.gov/removal-statistics/, consulté le 8 octobre 2015.

United States. President's Commission on Immigration and Naturalization, Whom We Shall Welcome, Report, Washington, D.C., U.S. Government Printing Office, 1953.

Wang, Yanan, “Donald Trump's 'Humane' 1950s Model for Deportation”, 'Operation Wetback', was Anything But", Washington Post, 30 septembre 2015.

\section{NOTES}

1. À noter que ce titre n'est pas une question. Il est tiré d'une citation de George Washington (1783) invitant l'Amérique à recevoir point seulement « l'étranger opulent et respectable » mais aussi les «persécutés de toutes les nations et religions ». Whom We Shall Welcome: Report of the President's Commission on Immigration and Naturalization, Washington, DC, Superintendent of Documents, U.S. Government Printing Office, 1953, page de garde.

2. La définition du terme "réfugié" n'évolua pour prendre un sens plus large que dans les années 1980.

3. Sur le statut légal des immigrés en situation irrégulière voir Motomura H., 2014.

4. Avec le soutien de la police et de l'armée, l'Immigration and Naturalization Service avait reconduit de force à l'intérieur des terres mexicaines près d'un million d'immigrés mexicains en situation irrégulière, surnommés «Wetbacks » en raison du fréquent passage clandestin par le Rio Grande.

\section{AUTEUR}

\section{BÉNÉDICTE DESCHAMPS}

Maître de conférences à l'Université Paris Diderot - Université Sorbonne Paris Cité- (LARCA UMR 8225), Bénédicte Deschamps a publié, avec Dominique Daniel, L'immigration aux États-Unis de 1607 à nos jours, Ellipses, 1998. Elle a récemment dirigé l'ouvrage collectif Immigration et travail aux États-Unis, publié en 2013 chez L'Harmattan. 